\title{
Estimation de la fiabilité par les essais accélérés
}

\author{
Ouahiba Tebbi, Fabrice Guérin ${ }^{\mathrm{a}}$ et Bernard Dumon
}

LASQUO EA 3858 : Laboratoire en Sûreté de fonctionnement, Qualité et Organisation, Institut des Sciences et Techniques de l'Ingénieur d'Angers, 62 avenue Notre Dame du Lac, 49000 Angers, France

Reçu le 9 janvier 2004, accepté le 5 mars 2004

Résumé - Dans cet article nous présentons quelques plans d'essais accélérés pour estimer la fiabilité des produits. Les plans étudiés sont basés sur le modèle standard de vie accélérée (SVA) en considérant des estimations paramétrique et non-paramétrique. Des applications sur des trombones, soumis à de la fatigue oligocyclique, sont présentées pour illustrer ces différents plans.

Mots clés : Fiabilité / estimation paramétrique / estimation non-paramétrique / dommage par fatigue / Miner, Basquin

\begin{abstract}
Reliability assessment by accelerated testing. In this paper various accelerated test plans are presented to assess the product reliability. The studied test plans are based on standard accelerated life model in assuming the parametric and nonparametric estimations. The paper is illustrated by an applying on clip paper subjected to low fatigue.
\end{abstract}

Key words: Reliability / parametric estimation / nonparametric estimation / fatigue damage / Miner / Basquin model

\section{Introduction}

Les essais accélérés sont une des approches les plus communes pour obtenir la loi de fiabilité ou le taux de défaillance de systèmes ou de composants dans des délais plus courts. Brièvement, ces essais consistent à réduire les durées de vie des produits par l'accélération des dégradations provoquant la défaillance. Pour ce faire, les niveaux des stress subis par le produit sont sévérisés afin d'obtenir des données plus rapidement. Pour obtenir la loi de fiabilité dans les conditions nominales, il est nécessaire d'utiliser un modèle de vie accélérée permettant de la définir à partir des résultats d'essais accélérés.

Il existe différentes classes (voir Fig. 1) de modèles de vie accélérée :

1. Les modèles expérimentaux déterminés par des plans d'expériences [1].

2. Les modèles physiques définis à l'aide de ceux de dégradation (chimique, mécanique, ... ) [2-4].

3. Les modèles statistiques caractérisés par des approches paramétrique, semi-paramétrique et nonparamétrique [5-21].

\footnotetext{
a Auteur correspondant :

fabrice.guerin@istia.univ-angers.fr
}

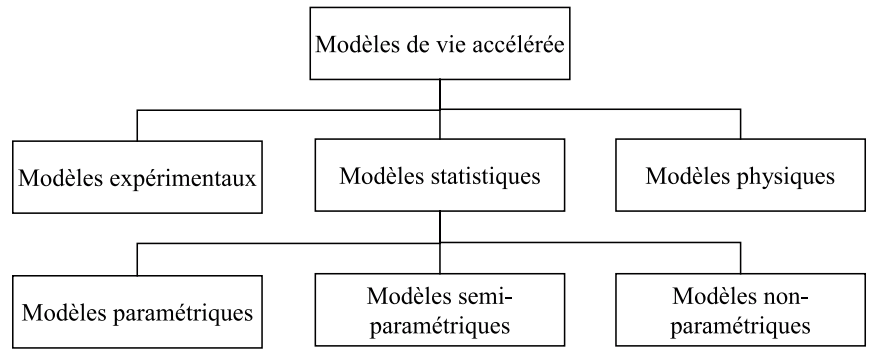

Fig. 1. Classification des modèles de vie accélérée.

On trouve une littérature abondante d'application des modèles de vie accélérée dans le domaine de l'électronique $[5,11,15,19]$ mais moins nombreuse dans celui de la mécanique $[2-5,16-18]$.

Aussi, nous proposons dans cet article d'étudier les modèles standards de vie accélérée (SVA) appliqués à la mécanique et plus particulièrement aux composants soumis au dommage par fatigue. Nous indiquons la signification mécanique de la fonction de transfert des SVA. Ensuite, nous étudierons 2 types de plan d'essais :

- Les plans d'essais accélérés prédictifs.

- Les plans d'essais avec endommagement accéléré préalable. 


\section{Nomenclature}

\begin{tabular}{|c|c|}
\hline$s$ & vecteur de stress utilisés en conditions sévérisées \\
\hline$s_{0}$ & vecteur de stress en conditions nominales \\
\hline$R_{s}(t)$ & fiabilité à l'instant $t$ sous stress $s$ \\
\hline$R_{s 0}(t)$ & fiabilité à l'instant $t$ sous stress nominal $s_{0}$ \\
\hline$f_{s}(t)$ & fonction de transfert permettant de passer de $R_{s}$ à $R_{s 0}$ \\
\hline$r(s)$ & facteur d'accélération permettant de passer des conditions sévérisées à celles nominales \\
\hline$\rho\left(s_{1}, s_{2}\right)$ & facteur d'accélération permettant de passer des conditions sévérisées $s_{1}$ à $s_{2}$ \\
\hline$\delta(s)$ & taux de changement d'échelle entre les conditions $s_{1}$ et $s_{2}$ \\
\hline$\beta$ & paramètres du modèle d'accélération \\
\hline$z$ & le vecteur de primitives \\
\hline$N$ & nombre de cycles à rupture \\
\hline$R_{m}$ & limite à la rupture \\
\hline$\sigma_{\mathrm{a}}$ & contrainte d'amplitude \\
\hline$\sigma_{D}$ & limite d'endurance \\
\hline$d$ & taux d'endommagement \\
\hline$D$ & endommagement cumulé \\
\hline$n$ & nombre de cycle \\
\hline$\phi$ & fonction de répartition de la loi normale standard \\
\hline$\dot{d}$ & vitesse d'endommagement \\
\hline$k, A$ & paramètres du modèle de Basquin \\
\hline$\gamma$ & paramètre de la loi d'accélération \\
\hline$\theta$ & paramètre d'échelle de la loi de fiabilité \\
\hline$v$ & paramètre de forme de la loi de fiabilité \\
\hline$\sigma$ & écart type \\
\hline$\mu$ & moyenne \\
\hline$L\left(x_{1}, x_{2}\right)$ & vraisemblance de paramètres $x_{1}$ et $x_{2}$ \\
\hline$\eta$ & logarithme décimal de $N$ \\
\hline$S(\eta)$ & nombre de systèmes survivants à l'instant $\eta$ \\
\hline$K(\eta)$ & nombre de systèmes défaillants à l'instant $\eta$ \\
\hline$\Delta K(\eta)$ & nombre de systèmes défaillants entre l'instant $\eta-$ et $\eta$ \\
\hline$F$ & fonction de répartition de la loi normale \\
\hline$u$ & variable centrée réduite \\
\hline
\end{tabular}

Dans les 2 cas, nous présenterons les estimations de fiabilité paramétrique et non-paramétrique.

\section{Modèle standard de vie accélérée}

Nous allons présenter le modèle standard de vie accélérée (SVA) basé sur la définition d'une fonction de transfert qui a été décrite par Bagdonavicius et Nikulin $[6,10,22,23]$.

Supposons que l'on ait des systèmes très fiables ne permettant pas d'observer des défaillances pendant un temps $t$ donné d'essai. Pour permettre la construction d'une loi de fiabilité, il est nécessaire de procéder par des essais accélérés au cours desquels les systèmes subissent des stress supérieurs à ceux qu'ils supportent dans les conditions normales d'utilisation.
Dans le cas général, les stress $s$ peuvent évoluer en fonction du temps et peuvent être multidimensionnels :

$$
s=s(\tau), \tau \geq 0 \text { où } s:[0, \infty[
$$

Supposons que la durée de vie $T_{s(.)}$ d'un produit sous le stress constant $s($.$) soit une variable aléatoire de fonc-$ tion de survie :

$$
R_{s(.)}(t)=\operatorname{prob}\left(T_{s(.)}>t\right), \quad t \geq 0
$$

Soit $R_{s 0}$ la fiabilité sous le stress usuel : $s_{0} \in \varepsilon_{0} \subset \varepsilon$ ( $\varepsilon$ Ensemble de stress), $R_{s 0}^{-1}$ sa fonction inverse.

La fonction de transfert est définie par :

$$
\begin{gathered}
f:[0,+\infty[\times \varepsilon \rightarrow[0,+\infty[ \\
(t, s(.)) \rightarrow f(t, s(.))=\left(R_{s_{0}}^{-1} \circ R_{s(.)}\right)(t)
\end{gathered}
$$




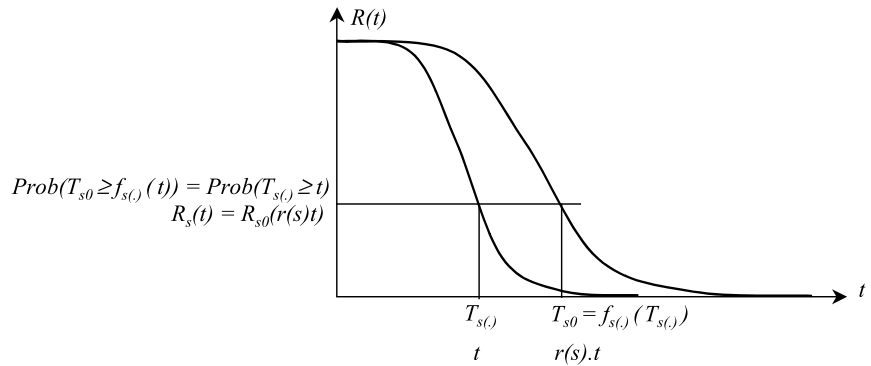

Fig. 2. Définition de la fonction de transfert.

Cette fonction permet d'évaluer la fiabilité d'un produit, sous des stress expérimentalement indisponibles, à partir de la fiabilité du même produit sous des conditions particulières d'utilisation.

La définition de $f$ peut être traduite par l'équation suivante :

$$
\operatorname{Prob}\left(T_{s_{0}} \geq f_{s(.)}(t)\right)=\operatorname{Prob}\left(T_{s(.)} \geq t\right)
$$

$f_{s(.)}(t)$ est appelée ressource utilisée sous le stress $s($. jusqu'à l'instant $t$.

Le modèle statistique de vie accélérée est défini sur $\varepsilon$ s'il existe une fonction $r: \varepsilon \rightarrow R^{+}$telle que pour tout $s(.) \in \varepsilon$ :

$$
\frac{\mathrm{d}}{\mathrm{d} t} f_{s(.)}(t)=r[s(t)]
$$

L'équation (4) implique :

$$
R_{\mathrm{S}(.)}(t)=R_{s_{0}}\left(\int_{0}^{t} r[s(\tau)] \mathrm{d} \tau\right)
$$

Dans le cas où $s(t)$ est constant l'équation (5) devient :

$$
R_{s}(t)=R_{s_{0}}(r(s) . t)
$$

On note que $r\left(s_{0}\right)=1$.

Considérons un plan d'essai classique consistant à utiliser plusieurs niveaux de stress constant $s_{1}, s_{2}, \ldots, s_{k}$ : $s_{1}<s_{2}<\ldots<s_{k}$ et $s_{0}\left(s_{0}<s_{1}\right)$ le stress usuel qui ne sera pas utilisé. Si la fonction $r$ est complètement inconnue alors la fonction de fiabilité $R_{s 0}$ ne peut être déterminée. Donc, il est nécessaire de choisir $r$ dans une certaine classe de fonctions. Bagdonavicius et Nikulin considèrent une forme exponentielle. Si l'équation (6) est vérifiée sur un ensemble de stress $\varepsilon$, alors pour tous $s_{1}$, $s_{2} \in \varepsilon_{0}$ :

$$
R_{s 2}(t)=R_{s 1}\left(\rho\left(s_{1}, s_{2}\right) t\right)
$$

où $\rho\left(s_{1}, s_{2}\right)=r\left(s_{2}\right) / r\left(s_{1}\right)$.

Supposons dans un premier temps que le stress $s \in$ $\varepsilon_{0} \subset \mathbb{R}$ soit unidimensionnel. Le taux de changement d'échelle est déterminé par la dérivée :

$$
\delta(s)=\lim _{\Delta s \rightarrow 0} \frac{\rho(s, s+\Delta s)-\rho(s, s)}{\Delta s}=\frac{\operatorname{dlog}(r(s))}{\mathrm{d} s}
$$

Donc pour tout $s \in \varepsilon$

$$
r(s)=e^{\int_{s_{0}}^{s} \delta(v) \mathrm{d} v}
$$

Supposons que $\delta(s)$ soit proportionnelle à une fonction connue $u(s)$ du stress :

$$
\delta(s)=\beta_{1} u(s), \quad \beta_{1}>0
$$

Alors

$$
r(s)=e^{\beta_{0}+\beta_{1} z(s)}
$$

où $z(s)$ est une fonction primitive de $u(s), \beta_{0}$ et $\beta_{1}$ sont des paramètres inconnus.

Par exemple, si on considère $\delta(s)=\beta_{1} / s$ alors on obtient le modèle de puissance inverse :

$$
r(s)=e^{\beta_{0}+\beta_{1} \log (s)}=\alpha s^{\beta_{1}}
$$

avec $\alpha=e^{\beta 0}$.

Ce modèle est appliqué dans le cas de système soumis au phénomène mécanique de fatigue.

Ainsi, les expressions (5) et (6) s'écrivent :

$$
R_{\mathrm{S}(.)}(t)=R_{s_{0}}\left(\int_{0}^{t} e^{\beta^{T} z(\tau)} \mathrm{d} \tau\right)
$$

$\mathrm{Ou}$

$$
R_{s}(t)=R_{s_{0}}\left(e^{\beta^{T} z} \cdot t\right)
$$

où $\beta=\left(\beta_{0}, \ldots, \beta_{m}\right)^{T}$ est le vecteur de paramètres et $z(t)=\left(z_{0}(t), \ldots, z_{m}(t)\right)$ le vecteur de primitives (avec $\left.z_{0}=1\right)$.

Dans les sections suivantes, nous allons préciser l'application des modèles SVA à la mécanique et plus particulièrement aux systèmes soumis à l'endommagement par fatigue.

\section{Application des modèles SVA à la mécanique}

\subsection{Introduction}

Les modèles SVA peuvent s'appliquer à plusieurs domaines comme celui du vivant (sciences médicales), de l'électronique, ... et de la mécanique. Ce qui différencie les diverses applications ce sont les lois de fiabilité utilisées, les stress employés pour sévériser les essais et la nature de la fonction $r$ des modèles (13) et (14).

Ainsi, en mécanique il est courant de définir la loi de fiabilité par une distribution de type Weibull, log-normale ou Birnbaum-Saunders qui caractérisent correctement les durées de vie des systèmes soumis à des dégradations mécaniques $[18,20]$. La défaillance constatée est bien souvent une rupture de pièces mais les mécanismes d'endommagement sous-jacent peuvent être de nature totalement différente. Cela dépend essentiellement des conditions d'utilisation du système et des stress qui y seront appliqués. En effet, la combinaison de la température, la corrosion, un chargement cyclique, ... agit différemment selon le niveau de chacun des stress en provoquant des mécanismes de dégradation différents (fluage, fatiguecorrosion, fatigue, ....). Aussi, les mécaniciens ont l'habitude de définir des modèles de dégradation en fonction 
des stress prépondérants pour caractériser la durée de vie des systèmes. Nous proposons dans la suite de l'article d'appliquer les modèles SVA à la mécanique en intégrant les spécificités de la mécanique. À titre d'illustration, nous prendrons comme exemple d'application l'endommagement par fatigue.

\section{2 Étude des SVA en mécanique}

En considérant l'application des SVA à la fatigue dans le domaine d'endurance limitée [24], la fonction de fiabilité $R_{s 0}(n)$, avec $n$ représentant le nombre de cycle, est définie par une loi log-normale :

$$
R_{s 0}(n)=1-\phi\left(\ln \left(\frac{n}{\theta}\right)^{\nu}\right)
$$

avec $\phi$ la loi normale standard.

Alors, pour un stress constant $s$, la loi de fiabilité, définie par la relation (14), s'écrit :

$$
\begin{aligned}
R_{s}(n) & =1-\phi\left(\ln \left(\frac{r(s) \cdot n}{\theta}\right)^{\nu}\right) \\
& =1-\phi\left(\ln \left(\frac{e^{\beta^{T} z} \cdot n}{\theta}\right)^{\nu}\right)=1-\phi\left(\frac{\ln (t)-\gamma^{T} z}{\sigma}\right)
\end{aligned}
$$

où $\gamma=\left(\gamma_{0}, \ldots, \gamma_{m}\right), \gamma_{0}=\ln (\theta)-\beta_{0}, \gamma_{i}=-\beta_{i}$ et $\nu=1 / \sigma$.

Rappelons que le modèle SVA est vérifié s'il existe une fonction $r$ telle que :

$$
\frac{\mathrm{d}}{\mathrm{d} t} f_{s(.)}(t)=r[s(t)]
$$

La fonction $r$ représente physiquement le taux d'accroissement de la vitesse de dégradation. Dans le cas de la fatigue, cette fonction peut être déduite de l'endommagement défini par le critère de Miner [24-27].

Ainsi, pour respecter l'équivalence d'endommagement cumulé $\left(d_{1}=d_{0}\right.$, voir Fig. 3$)$ sous conditions accélérées $\sigma_{\mathrm{a} 1}$ et nominales $\sigma_{\mathrm{a} 0}$, la fonction $r$ doit vérifier :

$$
d_{1}=\frac{n}{N_{1}} d_{0}=\frac{r \cdot n}{N_{0}}
$$

d'où

$$
r=\frac{N_{0}}{N_{1}}
$$

Si on considère le modèle de Basquin [24-27] pour caractériser la courbe de Wöhler dans le domaine de fatigue limitée, l'endommagement s'écrit :

$$
d\left(\sigma_{a}\right)=\frac{n}{A\left(\sigma_{a}\right)^{-k}}
$$

d'où la vitesse d'endommagement définie par

$$
\dot{d}\left(\sigma_{a}\right)=\frac{1}{A}\left(\sigma_{a}\right)^{k}
$$

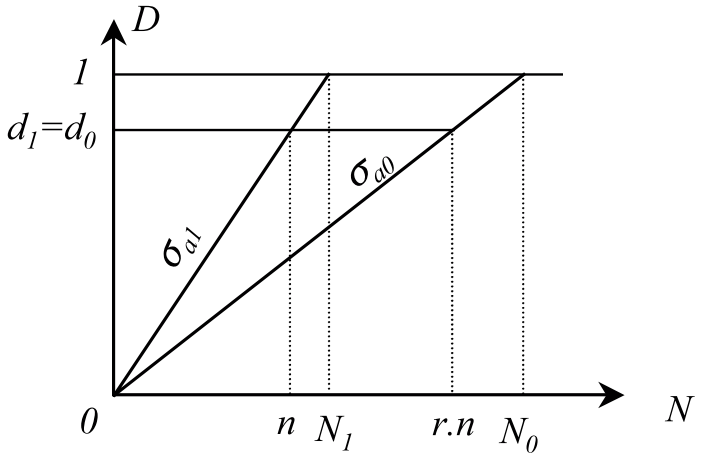

Fig. 3. Fonction de transfert dans le cas de la fatigue.

Ainsi, la relation (22) s'écrit :

$$
r\left(\sigma_{\mathrm{a} 1}\right)=\left(\frac{\sigma_{\mathrm{a} 1}}{\sigma_{\mathrm{a} 0}}\right)^{k}=\frac{\dot{d}\left(\sigma_{\mathrm{a} 1}\right)}{\dot{d}\left(\sigma_{\mathrm{a} 0}\right)}
$$

Pour faire l'analogie avec l'interprétation de Bagdonavicius et Nikulin [22] à propos de $r$, nous pouvons dire que ce terme correspond au taux d'accroissement de la vitesse d'endommagement sous conditions accélérées par rapport aux conditions nominales.

Ainsi, la loi de fiabilité $R_{\sigma_{\mathrm{a} 1}}(n)$, définie par la relation (15), s'écrit :

$R_{\sigma_{\mathrm{a} 1}}(n)=1-\phi\left(\ln \left(\frac{r\left(\sigma_{\mathrm{a} 1}\right) \cdot n}{N_{0}}\right)^{\nu}\right)=1-\phi\left(\ln \left(\frac{n}{N_{1}}\right)^{\nu}\right)$

\subsection{Plan d'essai accéléré prédictif}

\subsubsection{Définition du plan d'essai}

Ce plan consiste à déterminer les paramètres de la fonction $r(s)$ à partir de résultats d'essais effectués uniquement dans des conditions sévérisées et de déduire par régression la loi de fiabilité en conditions nominales (Fig. 4).

Ainsi, on réalise des essais dans les conditions sévérisées $s_{1}, s_{2}, \ldots, s_{n}$ et on déduit les paramètres $\beta$ du modèle (14) permettant de caractériser la loi de fiabilité $R_{s 0}(t)$.

Pour illustrer ce premier plan d'essai, nous allons considérer 3 types d'estimation de la fiabilité :

- paramétrique [5, 6, 8, 11, 15, 20,22],

- non-paramétrique [12,21,22].

\subsubsection{Application des SVA paramétriques}

L'estimation paramétrique d'un modèle SVA consiste à prendre une loi statistique particulière pour caractériser la fiabilité $[5,6,8,11,15,20,22]$. Dans le cas de la fatigue, la loi plus appropriée pour caractériser la fiabilité 


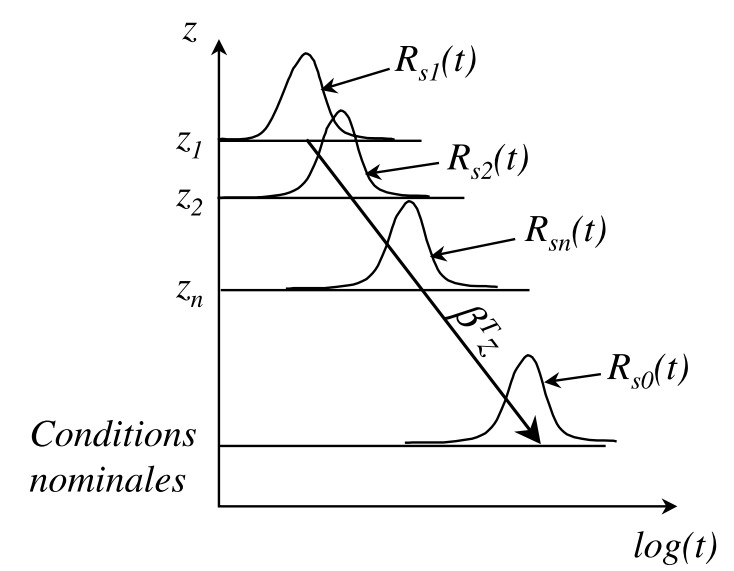

Fig. 4. Définition du plan d'essai par régression.

est la loi normale (lorsque l'on considère la distribution $\operatorname{des} \log (N))$ :

$$
R_{s_{0}}(N)=1-\phi\left(\frac{\log (N)-\mu}{\sigma}\right)
$$

où $\phi$ représente la fonction de répartition de la loi normale standard, $\mu$ la moyenne, $\sigma$ l'écart type.

Considérons un essai dans lequel on fixe la durée maximale d'expérience $N_{i}$ (exprimé en nombre de cycle pour la fatigue) du ième échantillon (de taille $n_{i}$ ) sous stress sévérisé $s_{i}$ (avec $i=1, \ldots, k$ et $\left.z^{(i)}=z_{i 0}, \ldots, z_{i m}\right)$ et $m_{i}$ le nombre de défaillances.

Donc le modèle (14) devient :

$$
R_{s}(N)=1-\phi\left(\frac{\log (N)-\gamma^{T} z}{\sigma}\right) \text { avec } t>0
$$

où $\gamma=\left(\gamma_{0}, \ldots, \gamma_{m}\right)$ et $\gamma_{0}=\log (\mu)-\beta_{0}$.

La vraisemblance est définie par :

$$
\begin{aligned}
L(\gamma, \sigma)=\prod_{i=1}^{k} & \prod_{j=1}^{m_{i}} \frac{1}{\sigma \sqrt{2 \pi}} e^{-\frac{1}{2}\left(\frac{\log \left(N_{i j}\right)-\gamma^{T} z^{(i)}}{\sigma}\right)^{2}} \\
& \times\left(1-\phi\left(\frac{\log \left(N_{i}\right)-\gamma^{T} z^{(i)}}{\sigma}\right)\right)^{n_{i}-m_{i}}
\end{aligned}
$$

avec $N_{i j}$ les nombres de cycles à rupture sous le stress $s_{i}$ ( $j$ ième défaillance), $N_{i}$ le nombre de cycles de la censure sous le stress $s_{i}$.

Par le maximum de vraisemblance [2], nous déduisons des estimateurs de $\hat{\gamma}$ et $\hat{\sigma}$ permettant de définir la loi de fiabilité dans les conditions nominales :

$$
R_{s_{0}}(N)=1-\phi\left(\frac{\log (N)-\hat{\gamma}^{T} z^{(0)}}{\hat{\sigma}}\right) \text { avec } N>0
$$

où $z^{(0)}$ représente le vecteur de stress dans les conditions nominales.

\subsubsection{Application des SVA non-paramétriques}

Ce dernier cas consiste à ne faire aucune hypothèse de loi de fiabilité $[12,21,22]$. Pour ce faire, on construit la loi de fiabilité à l'aide de l'estimateur de Kaplan-Meier. Notons $K_{i}(\eta)$ le nombre de défaillances observées du $i$ ème échantillon dans l'intervalle $[0, \eta]$, et $S_{i}(\eta)$ le nombre de survivants avant le nombre de cycles $\eta$. $N_{i 1}, \ldots, N_{i m i}$ représentent les nombres de cycles à rupture du ième échantillon sous stress $s_{i}\left(\operatorname{avec} m_{i}=K_{i}\left(\log \left(N_{i}\right)\right)\right.$. Les variables aléatoires :

$$
e^{\beta^{T} z_{i}} \log \left(N_{i j}\right) \quad\left(i=1, \ldots, k ; j=1, \ldots, m_{i}\right)
$$

peuvent être considérées comme des pseudo-ruptures observées dans un essai où $n=\sum_{i=1}^{k} n_{i}$ pièces avec la loi de fiabilité $R_{s 0}$ ont été testées et $n_{i}-m_{i}$ parmi elles ont été censurées au moment $e^{\beta^{T} z_{i}} N_{i}$.

Soit le nombre total de défaillances dans l'intervalle $[0, \eta]$ :

$$
K(\eta, \beta)=\sum_{i=1}^{k} K_{i}\left(e^{-\beta^{T} z_{i}} \eta\right)
$$

et le nombre total de survivants avant l'instant $\eta$ :

$$
S(\eta, \beta)=\sum_{i=1}^{k} S_{i}\left(e^{-\beta^{T} z_{i}} \eta\right)
$$

La loi de fiabilité peut être construite par l'estimateur de Kaplan-Meier : pour tous $x \leq \max _{i}\left(e^{\beta^{T} z_{i}} \log \left(N_{i}\right)\right)$

$$
\begin{aligned}
R_{s_{0}}(x, \beta) & =\prod_{\eta \leq x}\left(1-\frac{\Delta K(\eta, \beta)}{S(\eta, \beta)}\right) \\
& =\prod_{\eta \leq x}\left(1-\frac{\sum_{j=1}^{k} \Delta K_{j}\left(e^{-\beta^{T} z_{j}} \eta\right)}{\sum_{j=1}^{k} S_{j}\left(e^{-\beta^{T} z_{j}} \eta\right)}\right)
\end{aligned}
$$

où $\Delta K(\eta, \beta)=K(\eta, \beta)-K(\eta-, \beta)$ et $\Delta K_{j}(\eta, \beta)=$ $K_{j}(\eta)-K_{j}(\eta-)$.

La fonction de vraisemblance est définie par :

$$
\begin{aligned}
L(\beta)= & \prod_{i=1}^{k} \prod_{j=1}^{m_{i}}\left[R_{s_{0}}\left(e^{\beta^{T} z_{i}} \log \left(N_{i j}-\right), \beta\right)\right. \\
& \left.-R_{s_{0}}\left(e^{\beta^{T} z_{i}} \log \left(N_{i j}\right), \beta\right)\right] \\
& \times\left(R_{s_{0}}\left(e^{\beta^{T} z_{i}} \log \left(N_{i}\right), \beta\right)\right)^{n_{i}-m_{i}}
\end{aligned}
$$

en approximant la fonction densité $f_{s 0}=-R_{s 0}^{\prime}$ inconnue par :

$$
R_{s_{0}}\left(e^{\beta^{T} z_{i}} \log \left(N_{i j}-\right), \beta\right)-R_{s_{0}}\left(e^{\beta^{T} z_{i}} \log \left(N_{i j}\right), \beta\right)
$$

Par le maximum de vraisemblance, nous obtenons une estimation de $\hat{\beta}$ permettant de construire la loi de fiabilité dans les conditions nominales. 


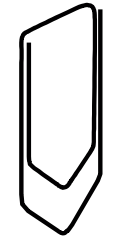

(a)

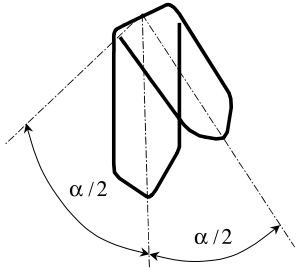

(b)

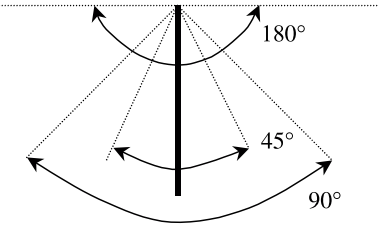

(c)

Fig. 5. (a) Trombone, (b) déformation du trombone, (c) les 3 niveaux de déformation.

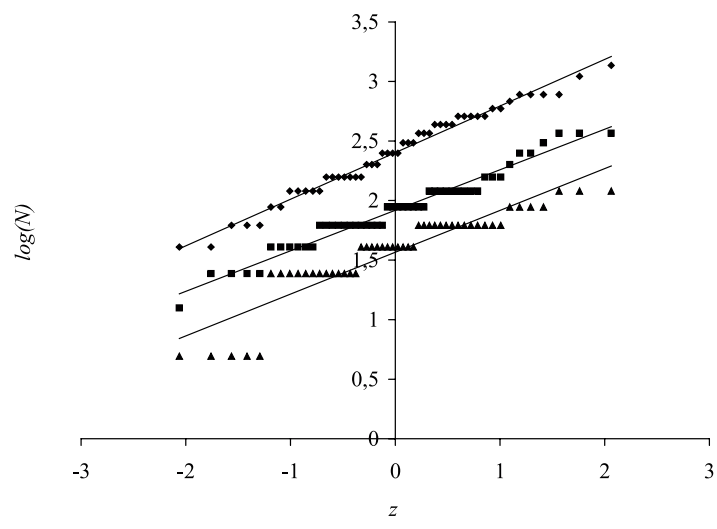

Fig. 6. Les droites d'Henry.

\subsubsection{Exemple expérimental}

Dans cet exemple (proposé par Vassiliou [15]), nous considérons un trombone (Fig. 5a). L'objectif est de définir la fiabilité, en fonction du nombre de cycles à rupture (phénomène de fatigue oligocyclique, voir Fig. 4), du trombone sous déformation angulaire. Les conditions nominales sont supposées être une déformation angulaire de $45^{\circ}$ (Fig. 5b). L'estimation de la fiabilité se fera en réalisant un essai accéléré sous 2 conditions sévérisées correspondant aux déformations angulaire de $90^{\circ}$ et $180^{\circ}$ (Fig. 5c).

Les trombones sont testés sous 3 angles d'ouverture de $45^{\circ}, 90^{\circ}$ et $180^{\circ}$. Les nombres de cycles à rupture sont enregistrés (voir Tab. 1).

Les résultats sont analysés en supposant une distribution normale des $\log (N)$. Ainsi, pour les trois séries d'essais sont tracées les droites d'Henry $(\log (N)=g(u))$ associées (Fig. 6).

Les moyennes et écart types sont évalués pour chaque série d'essai :

$$
\begin{array}{lll}
\mu^{\left(45^{\circ}\right)}=2,4 & \mu^{\left(90^{\circ}\right)}=1,92 & \mu^{\left(180^{\circ}\right)}=1,56 \\
\sigma^{\left(45^{\circ}\right)}=0,37 & \sigma^{\left(90^{\circ}\right)}=0,33 & \sigma^{\left(180^{\circ}\right)}=0,36
\end{array}
$$

Des droites d'Henry parallèles, l'hypothèse de constance de l'écart type sous les différents niveaux de stress est vérifiée. Dans la suite, nous analysons les résultats d'essai accélérés par les modèles SVA suivants :

- paramétrique,

- non-paramétrique.

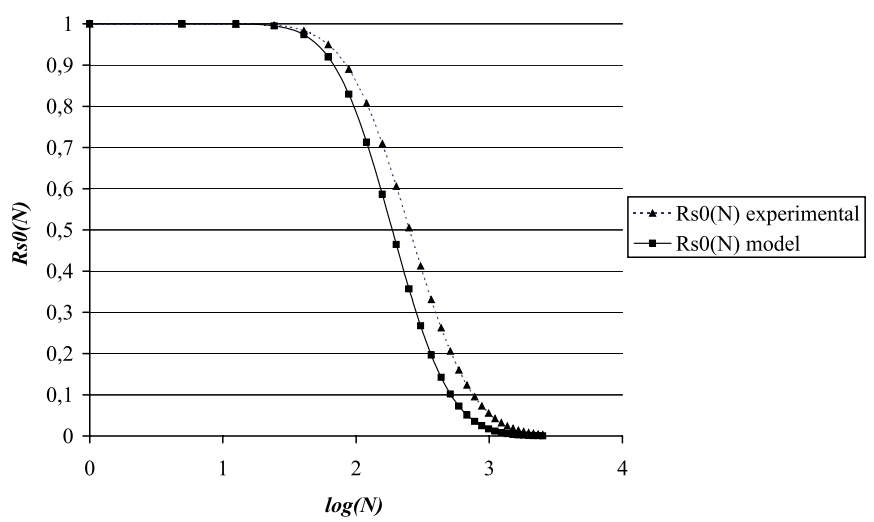

Fig. 7. Fonction fiabilité par un modèle SVA paramétrique.

\section{a) Application du modèle SVA paramétrique}

Le modèle de puissance inverse [5] est choisi pour caractériser la fonction $r(s)$ dépendant de l'angle $\alpha$ :

$$
r(\alpha)=e^{\beta_{0}+\beta_{1} \ln (\alpha)}
$$

En considérant une distribution normale des $\log (N)$ pour caractériser la fonction de fiabilité, le vecteur $\beta$ et l'écart type $\sigma$ sont obtenus par le maximum de vraisemblance (définie par l'Éq. (26)) :

$$
\hat{\beta}_{0}=1,835 \quad \hat{\beta}_{1}=-0,2654 \quad \hat{\sigma}=0,343
$$

La moyenne sous conditions nominales est évaluée par :

$$
\mu^{(0)}=r\left(45^{\circ}\right)=e^{1,835-0,2654 \times \log \left(45^{\circ}\right)}=2,28
$$

Alors, la fonction de fiabilité sous conditions nominales est définie (Fig. 7).

\section{b) Application d'un modèle SVA non-paramétrique}

Le modèle puissance inverse est utilisé pour définir la fonction fiabilité :

$$
e^{\beta^{T} z}=e^{\beta_{0}+\beta_{1} \ln (\alpha)}
$$

L'estimateur de Kaplan-Meier est utilisé pour définir la fonction de fiabilité sous conditions nominales (Éq. (30)). Après, le vecteur $\beta$ est estimé par le maximum de vraisemblance :

$$
\hat{\beta}_{0}=-1,0015 \quad \hat{\beta}_{1}=0,272
$$

Finalement, la fonction de fiabilité sous conditions nominales est obtenue (Fig. 8). 
Tableau 1. Résultats expérimentaux.

\begin{tabular}{|c|c|c|c|c|c|c|c|}
\hline$N-45^{\circ}$ & $\log \left(N-45^{\circ}\right)$ & $N-90^{\circ}$ & $\log \left(N-90^{\circ}\right)$ & $N-180^{\circ}$ & $\log \left(N-180^{\circ}\right)$ & $F \%$ & $u=\phi^{-1}(F \%)$ \\
\hline 5 & 1,609 & 3 & 1,099 & 2 & 0,693 & 0,020 & $-2,062$ \\
\hline 5 & 1,609 & 4 & 1,386 & 2 & 0,693 & 0,039 & $-1,760$ \\
\hline 6 & 1,792 & 4 & 1,386 & 2 & 0,693 & 0,059 & $-1,565$ \\
\hline 6 & 1,792 & 4 & 1,386 & 2 & 0,693 & 0,078 & $-1,416$ \\
\hline 6 & 1,792 & 4 & 1,386 & 2 & 0,693 & 0,098 & $-1,293$ \\
\hline 7 & 1,946 & 5 & 1,609 & 4 & 1,386 & 0,118 & $-1,187$ \\
\hline 7 & 1,946 & 5 & 1,609 & 4 & 1,386 & 0,137 & $-1,093$ \\
\hline 8 & 2,079 & 5 & 1,609 & 4 & 1,386 & 0,157 & $-1,007$ \\
\hline 8 & 2,079 & 5 & 1,609 & 4 & 1,386 & 0,176 & $-0,929$ \\
\hline 8 & 2,079 & 5 & 1,609 & 4 & 1,386 & 0,196 & $-0,856$ \\
\hline 8 & 2,079 & 5 & 1,609 & 4 & 1,386 & 0,216 & $-0,787$ \\
\hline 8 & 2,079 & 6 & 1,792 & 4 & 1,386 & 0,235 & $-0,722$ \\
\hline 9 & 2,197 & 6 & 1,792 & 4 & 1,386 & 0,255 & $-0,659$ \\
\hline 9 & 2,197 & 6 & 1,792 & 4 & 1,386 & 0,275 & $-0,599$ \\
\hline 9 & 2,197 & 6 & 1,792 & 4 & 1,386 & 0,294 & $-0,541$ \\
\hline 9 & 2,197 & 6 & 1,792 & 4 & 1,386 & 0,314 & $-0,485$ \\
\hline 9 & 2,197 & 6 & 1,792 & 4 & 1,386 & 0,333 & $-0,431$ \\
\hline 9 & 2,197 & 6 & 1,792 & 4 & 1,386 & 0,353 & $-0,377$ \\
\hline 9 & 2,197 & 6 & 1,792 & 5 & 1,609 & 0,373 & $-0,325$ \\
\hline 10 & 2,303 & 6 & 1,792 & 5 & 1,609 & 0,392 & $-0,274$ \\
\hline 10 & 2,303 & 6 & 1,792 & 5 & 1,609 & 0,412 & $-0,223$ \\
\hline 10 & 2,303 & 6 & 1,792 & 5 & 1,609 & 0,431 & $-0,173$ \\
\hline 11 & 2,398 & 6 & 1,792 & 5 & 1,609 & 0,451 & $-0,123$ \\
\hline 11 & 2,398 & 7 & 1,946 & 5 & 1,609 & 0,471 & $-0,074$ \\
\hline 11 & 2,398 & 7 & 1,946 & 5 & 1,609 & 0,490 & $-0,025$ \\
\hline 11 & 2,398 & 7 & 1,946 & 5 & 1,609 & 0,510 & 0,025 \\
\hline 12 & 2,485 & 7 & 1,946 & 5 & 1,609 & 0,529 & 0,074 \\
\hline 12 & 2,485 & 7 & 1,946 & 5 & 1,609 & 0,549 & 0,123 \\
\hline 12 & 2,485 & 7 & 1,946 & 5 & 1,609 & 0,569 & 0,173 \\
\hline 13 & 2,565 & 7 & 1,946 & 6 & 1,792 & 0,588 & 0,223 \\
\hline 13 & 2,565 & 7 & 1,946 & 6 & 1,792 & 0,608 & 0,274 \\
\hline 13 & 2,565 & 8 & 2,079 & 6 & 1,792 & 0,627 & 0,325 \\
\hline 14 & 2,639 & 8 & 2,079 & 6 & 1,792 & 0,647 & 0,377 \\
\hline 14 & 2,639 & 8 & 2,079 & 6 & 1,792 & 0,667 & 0,431 \\
\hline 14 & 2,639 & 8 & 2,079 & 6 & 1,792 & 0,686 & 0,485 \\
\hline 14 & 2,639 & 8 & 2,079 & 6 & 1,792 & 0,706 & 0,541 \\
\hline 15 & 2,708 & 8 & 2,079 & 6 & 1,792 & 0,725 & 0,599 \\
\hline 15 & 2,708 & 8 & 2,079 & 6 & 1,792 & 0,745 & 0,659 \\
\hline 15 & 2,708 & 8 & 2,079 & 6 & 1,792 & 0,765 & 0,722 \\
\hline 15 & 2,708 & 8 & 2,079 & 6 & 1,792 & 0,784 & 0,787 \\
\hline 15 & 2,708 & 9 & 2,197 & 6 & 1,792 & 0,804 & 0,856 \\
\hline 16 & 2,773 & 9 & 2,197 & 6 & 1,792 & 0,824 & 0,929 \\
\hline 16 & 2,773 & 9 & 2,197 & 6 & 1,792 & 0,843 & 1,007 \\
\hline 17 & 2,833 & 10 & 2,303 & 7 & 1,946 & 0,863 & 1,093 \\
\hline 18 & 2,890 & 11 & 2,398 & 7 & 1,946 & 0,882 & 1,187 \\
\hline 18 & 2,890 & 11 & 2,398 & 7 & 1,946 & 0,902 & 1,293 \\
\hline 18 & 2,890 & 12 & 2,485 & 7 & 1,946 & 0,922 & 1,416 \\
\hline 18 & 2,890 & 13 & 2,565 & 8 & 2,079 & 0,941 & 1,565 \\
\hline 21 & 3,045 & 13 & 2,565 & 8 & 2,079 & 0,961 & 1,760 \\
\hline 23 & 3,135 & 13 & 2,565 & 8 & 2,079 & 0,980 & 2,062 \\
\hline
\end{tabular}




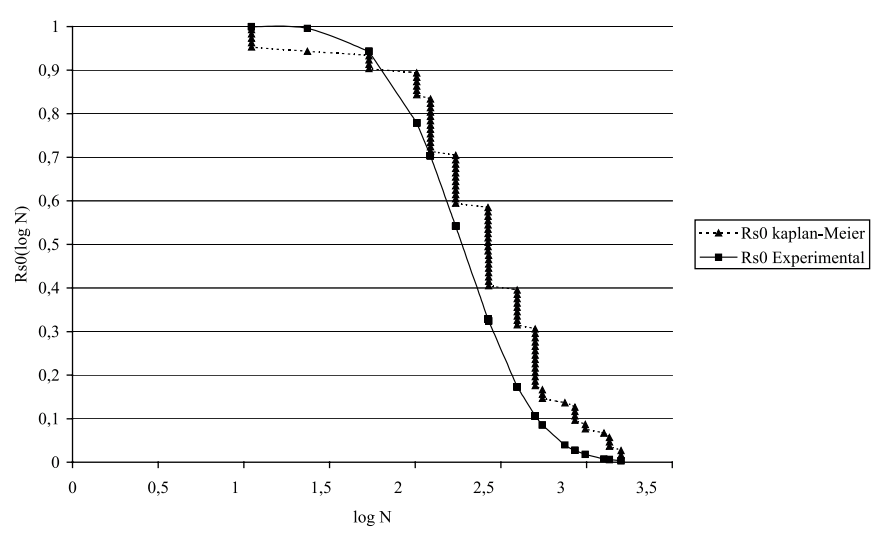

Fig. 8. Fonction de fiabilité par un modèle SVA nonparamétrique.

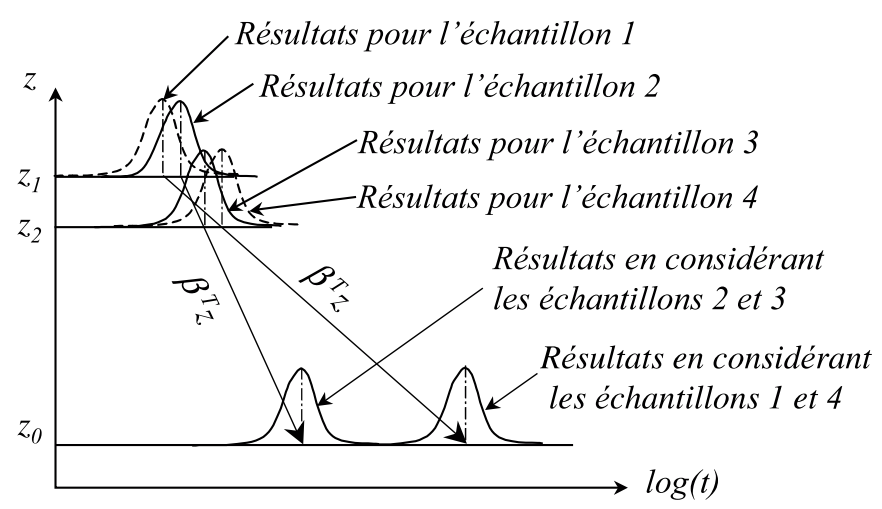

Fig. 9. Exemple de propagation d'incertitude.

\subsubsection{Analyse des résultats}

Nous constatons une bonne adéquation des modèles paramétriques et non-paramétriques avec les données expérimentales de référence $\left(\alpha=45^{\circ}\right)$ sur les trombones. Toutefois, ce type de plan d'essai par régression nécessite une bonne connaissance de la fonction $r(s)$ ce qui n'est pas toujours évident surtout dans les cas où de nombreuses variables sont utilisées pour sévériser les essais. Par conséquent, cela impose de réaliser des essais avec différentes combinaisons de niveaux de stress afin d'estimer les paramètres de $r(s)$. De plus, les niveaux de stress retenus pour réaliser les essais sont relativement élevés par rapport aux conditions nominales et les différentes estimations sont entachées d'incertitude. Cela provoque par régression une plus grande incertitude sur l'estimation de la loi de fiabilité dans les conditions nominales (voir Fig. 9).

\subsection{Plan d'essai avec endommagement préalable accéléré}

\subsubsection{Définition du plan d'essai}

Dans les paragraphes précédents, nous avons montré les difficultés de mener un essai accéléré par régression à l'aide des modèles classiques. Bagdonavicius et Nikulin

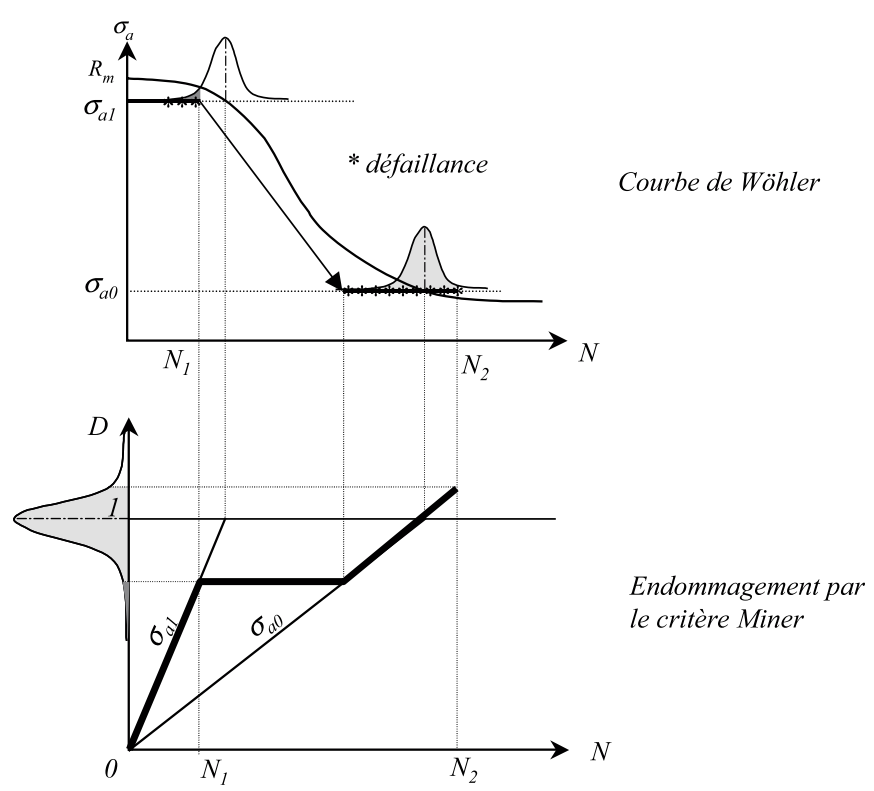

Fig. 10. Définition du plan d'essai avec endommagement préalable.

présentent une alternative en partant du principe que pour les systèmes hautement fiables la plupart des défaillances se produiront après un nombre de cycles $N_{2}$ importants. Il propose de procéder par un essai avec 2 groupes de composants. Le premier groupe sera testé sous un stress sévérisé $s_{1}$ et le deuxième sous un stress échelonné : sous le stress $s_{1}$ jusqu'au nombre de cycles $N_{1}<N_{2}$ et sous stress nominal $s_{0}$. Les pièces soumises au stress $s_{1}$ emmagasineront beaucoup d'endommagement jusqu'au nombre de cycles $N_{1}$ permettant d'obtenir des défaillances dans l'intervalle $\left[N_{1}, N_{2}\right]$.

Dans ce plan d'essai, il n'est pas nécessaire de paramétrer la fonction $r(s)$. Si le premier échantillon est testé sous le stress $s_{1}$ et le deuxième sous le stress :

$$
s_{2}(\eta)=\left\{\begin{array}{llc}
s_{1} & \text { si } & 0 \leq \eta \leq N_{1} \\
s_{0} & \text { si } & N_{1} \leq \eta \leq N_{2}
\end{array}\right.
$$

Alors le modèle (14) implique que

$$
R_{s_{1}}(\eta)=R_{s_{0}}(r \eta)
$$

et

$$
R_{s_{2}}(\eta)=\left\{\begin{array}{lc}
R_{s_{0}}(r \eta) & 0 \leq \eta \leq N_{1} \\
R_{s_{0}}\left(r \log \left(N_{1}\right)+\eta+\log \left(N_{1}\right)\right) & N_{1} \leq \eta \leq N_{2}
\end{array}\right.
$$

La figure suivante illustre la méthode (avec $f_{s 1}=$ $-R_{s 1}^{\prime}, f_{s 2}=-R_{s 2}^{\prime}$ et $\left.f_{s 0}=-R_{s 0}^{\prime}\right)$.

Les deux sections suivantes présentent l'application de ce plan d'essai dans les cas :

- paramétrique,

- non-paramétrique. 


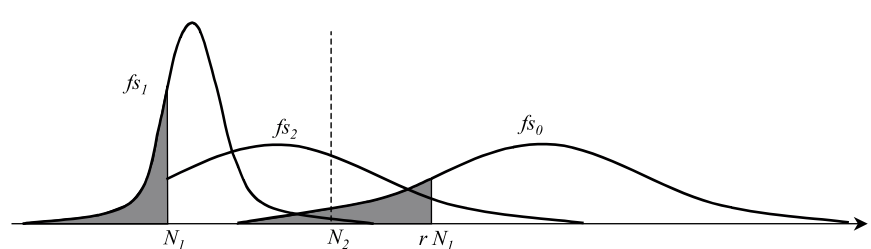

Fig. 11. Définition du plan d'essai par régression.

\subsubsection{Application du modèle SVA paramétrique}

Le premier échantillon de taille $n_{1}$ est testé sous stress sévérisé $s_{1}$ et on obtient les nombres de cycles à rupture : $N_{11}, \ldots, N_{1 n 1}$. Le deuxième échantillon, de taille $n_{2}$, est testé sous le stress échelonné suivant :

$$
s_{2}(\eta)=\left\{\begin{array}{llc}
s_{1} & \text { si } & 0 \leq \eta \leq N_{1} \\
s_{0} & \text { si } & N_{1} \leq \eta \leq N_{2}
\end{array}\right.
$$

Nous obtenons les nombres de cycles à rupture censurés à la date $N_{2}\left(N_{21}, \ldots, N_{2 m 2}, m_{2} \leq n_{2}\right)$ dont $k_{2}$ défaillances sous le stress $s_{1}$.

Supposons que les $\log (N)$ suivent une loi normale :

$$
R_{s_{0}}(\log (N))=1-\phi\left(\frac{\log (N)-\mu}{\sigma}\right)
$$

où $\phi$ représente la fonction de répartition de la loi normale standard, $\mu$ la moyenne, $\sigma$ l'écart type.

Donc le modèle (14) peut être écrit :

$$
R_{s}(\log (N))=R_{s_{0}}(r \cdot \log (N))=1-\phi\left(\frac{r \cdot \log (N)-\mu}{\sigma}\right)
$$

Ainsi, on obtient

$$
R_{s_{1}}(\log (N))=1-\phi\left(\frac{r \cdot \log (N)-\mu}{\sigma}\right)
$$

$$
\begin{aligned}
& R_{s_{2}}(\log (N))= \\
& \quad 1-\phi\left(\frac{r \cdot \log \left(N_{1}\right)+\log (N)-\log \left(N_{1}\right)-\mu}{\sigma}\right)
\end{aligned}
$$

La vraisemblance est définie par :

$$
\begin{aligned}
& L(r, \mu, \sigma)=\prod_{i=1}^{n_{1}} \frac{1}{\sigma \sqrt{2 \pi}} e^{-\frac{1}{2}\left(\frac{r \cdot \log \left(N_{1 i}\right)-\mu}{\sigma}\right)^{2}} \\
& \times \prod_{j=1}^{k_{2}} \frac{1}{\sigma \sqrt{2 \pi}} e^{-\frac{1}{2}\left(\frac{r \cdot \log \left(N_{2 j}\right)-\mu}{\sigma}\right)^{2}} \\
& \times \prod_{l=k_{2}+1}^{m_{2}} \frac{1}{\sigma \sqrt{2 \pi}} e^{-\frac{1}{2}\left(\frac{r \cdot \log \left(N_{1}\right)+\log \left(N_{2 l}\right)-\log \left(N_{1}\right)-\mu}{\sigma}\right)^{2}} \\
& \times\left(1-\phi\left(\frac{r \cdot \log \left(N_{1}\right)+\log \left(N_{2}\right)-\log \left(N_{1}\right)-\mu}{\sigma}\right)\right)^{n_{2}-m_{2}}
\end{aligned}
$$

Par le maximum de vraisemblance, nous déduisons des estimateurs de $\hat{r}, \hat{\mu}$ et $\hat{\sigma}$ permettant de définir la loi de fiabilité dans les conditions nominales :

$$
R_{s_{0}}(\log (N))=1-\phi\left(\frac{\log (N)-\hat{\mu}}{\hat{\sigma}}\right) \text { avec } N>0
$$

\subsubsection{Application du modèle SVA non-paramétrique}

Ce dernier cas consiste à ne faire aucune hypothèse de loi de fiabilité. Pour ce faire, on construit la loi de fiabilité à l'aide de l'estimateur de Kaplan-Meier.

Le premier échantillon de taille $n_{1}$ est testé sous stress sévérisé $s_{1}$ et on obtient les nombres de cycles à rupture : $N_{11}, \ldots, N_{1 n 1}$. Le deuxième échantillon, de taille $n_{2}$, est testé sous le stress $s_{1}$ jusqu'au nombre de cycles $N_{1}$ en obtenant $k_{2}$ ruptures et ensuite sous le stress $s_{0}$ jusqu'à $N_{2}$. Ainsi, nous obtenons les nombres de cycles à rupture censurés à la date $N_{2}\left(N_{21}, \ldots, N_{2 m 2}, m_{2} \leq n_{2}\right)$.

Notons :

- $K_{1}\left(\log \left(N_{1 i}\right) \leq \eta\right)$ le nombre de défaillances observées du premier échantillon dans l'intervalle $[0, \eta]$,

- $K_{2}\left(\log \left(N_{2 i}\right) \leq \eta\right)$ le nombre de défaillances observées du deuxième échantillon dans l'intervalle $[0, \eta]$,

- $S_{1}\left(\eta \geq \log \left(N_{1 i}\right)\right)$ le nombre de survivants du premier échantillon avant le nombre de cycles $\eta$,

- $S_{2}\left(\eta \geq \log \left(N_{2 i}\right)\right)$ le nombre de survivants du deuxième échantillon avant le nombre de cycles $\eta$ (y compris les $n_{2}-m_{2}$ systèmes censurés).

Le modèle (14) implique que

$R_{s 1}(\eta)=R_{s 0}(r . \eta)$

$$
\text { et }\left\{\begin{array}{rlrl}
R_{s_{2}}(\eta)= & R_{s_{0}}(r . \eta) & & \eta \leq \log \left(N_{1}\right) \\
R_{s_{2}}(\eta)= & R_{s_{0}}\left(r \cdot \log \left(N_{1}\right)\right. & \\
& \left.+\eta-\log \left(N_{1}\right)\right) & \eta \geq \log \left(N_{1}\right)
\end{array}\right.
$$

Les nombres de cycles $r . N_{2 i}$ et $r . N_{1 i}$ peuvent être interprétés comme des moments de défaillance obtenus dans un essai pendant lequel $n_{1}+n_{2}$ systèmes de fonction de fiabilité $R_{s 0}$ ont été observés et le nombre de cycles de la censure $N_{2}$ pour les systèmes du deuxième échantillon. Soit le nombre total de défaillances dans l'intervalle $[0, \eta]$ :

$$
\left\{\begin{array}{rlrl}
K(\eta)= & N_{1}\left(\frac{\eta}{r}\right)+N_{2}\left(\frac{\eta}{r}\right) & & \eta \leq \log (N 1) \\
K(\eta)= & N_{1}\left(\frac{\eta}{r}\right)+N_{2}(\log (N 1) & \\
& +\eta-r \cdot \log (N 1)) & & \eta>\log (N 1)
\end{array}\right.
$$

et le nombre total de survivants avant l'instant $\eta$ :

$$
\left\{\begin{array}{rlrl}
S(\eta)= & S_{1}\left(\frac{\eta}{r}\right)+S_{2}\left(\frac{\eta}{r}\right) & & \eta \leq \log (N 1) \\
S(\eta)= & S_{1}\left(\frac{\eta}{r}\right)+S_{2}(\log (N 1) & \\
& +\eta-r \cdot \log (N 1)) & & \eta>\log (N 1)
\end{array}\right.
$$


La loi de fiabilité peut être construite par l'estimateur de Kaplan-Meier :

$$
R_{s_{0}}(x, r)=\prod_{\eta \leq x}\left(1-\frac{\Delta K(\eta)}{S(\eta)}\right)
$$

où $\Delta K(\eta)=K(\eta)-K(\eta-)$ et $\Delta K(\eta)=K(\eta)-K(\eta-)$.

La fonction de vraisemblance est définie par :

$$
\begin{aligned}
& L(r)= \prod_{i=1}^{n_{1}}\left[R_{s_{0}}\left(r \cdot \log \left(N_{1 i}\right)-, r\right)-R_{s_{0}}\left(r \log \left(N_{1 i}\right), r\right)\right] \\
& \times \prod_{j=1}^{k_{2}}\left[R_{s_{0}}\left(r \log \left(N_{2 j}\right)-, r\right)-R_{s_{0}}\left(r \log \left(N_{2 j}\right), r\right)\right] \\
& \times \prod_{l=1+k_{2}}^{m_{2}}\left[R_{s_{0}}\left(\left[r \log \left(N_{1}\right)+\log \left(N_{2 l}\right)-\log \left(N_{1}\right)\right]-, r\right)\right. \\
&\left.\quad-R_{s_{0}}\left(\left[r \log \left(N_{1}\right)+\log \left(N_{2 l}\right)-\log \left(N_{1}\right)\right], r\right)\right] \\
& \quad \times\left[R_{s_{0}}\left(\left[r \log \left(N_{1}\right)+\log \left(N_{2}\right)-\log \left(N_{1}\right)\right], r\right)\right]^{n_{2}-m_{2}}
\end{aligned}
$$

en approximant la fonction densité $f_{s 0}=-R_{s 0}^{\prime}$ inconnue par :

$$
R_{s_{0}}(\eta-, r)-R_{s_{0}}(\eta, r)
$$

Par le maximum de vraisemblance, nous obtenons une estimation de $\hat{r}$ permettant de construire la loi de fiabilité dans les conditions nominales.

\subsubsection{Exemple expérimental}

Nous reprenons le cas du trombone (Fig. 5). L'estimation de la fiabilité se fera en réalisant un essai accéléré sur 2 échantillons. Le premier échantillon sera soumis à une déformation de $90^{\circ}$ et le deuxième à $90^{\circ}$ pendant 6 cycles et ensuite à une déformation de $45^{\circ}$ jusqu'à rupture (Fig. 16c). Un troisième échantillon est testé uniquement sous une déformation de $45^{\circ}$ servant de référence.

Les nombres de cycles à rupture sont enregistrés (voir Tab. 2).

Les valeurs en gras dans l'essai à « $90^{\circ}-45^{\circ}$ » représentent les résultats obtenus sous les stress à $90^{\circ}$ et les suivantes sous conditions nominales $\left(45^{\circ}\right)$ sans censure.

Les résultats sont analysés en supposant une distribution normale des $\log (N)$. Ainsi, pour les deux séries d'essais $\left(90^{\circ}\right.$ et $\left.45^{\circ}\right)$ sont tracées les droites d'Henry associées (Fig. 12).

Les moyennes et écart types sont évalués pour les 2 premiers tests :

$$
\begin{array}{ll}
\mu^{\left(45^{\circ}\right)}=2,4 & \mu^{\left(90^{\circ}\right)}=1,92 \\
\sigma^{\left(45^{\circ}\right)}=0,37 & \sigma^{\left(90^{\circ}\right)}=0,33
\end{array}
$$

Nous pouvons constater un parallélisme des droites d'Henry permettant de vérifier l'hypothèse écart type constant. Dans la suite, nous analysons les résultats d'essai accélérés par les modèles SVA suivants :

- paramétrique,

- non-paramétrique

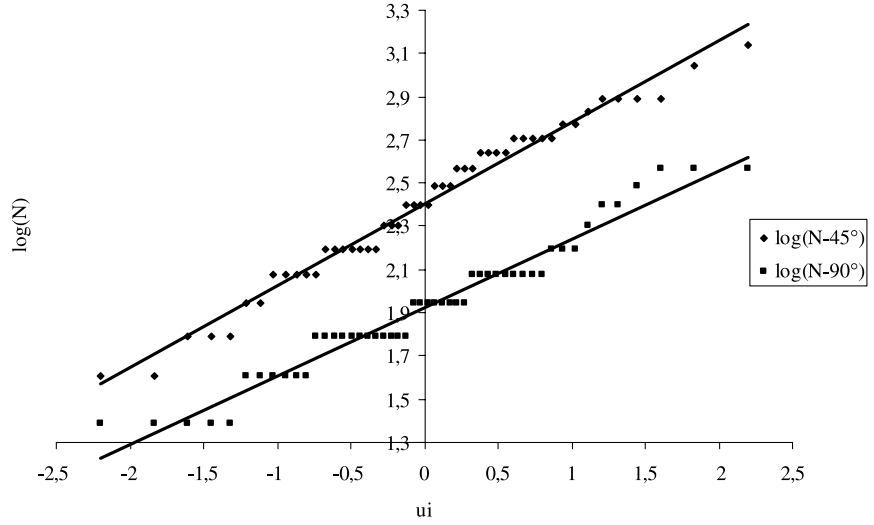

Fig. 12. Les droites d'Henry.

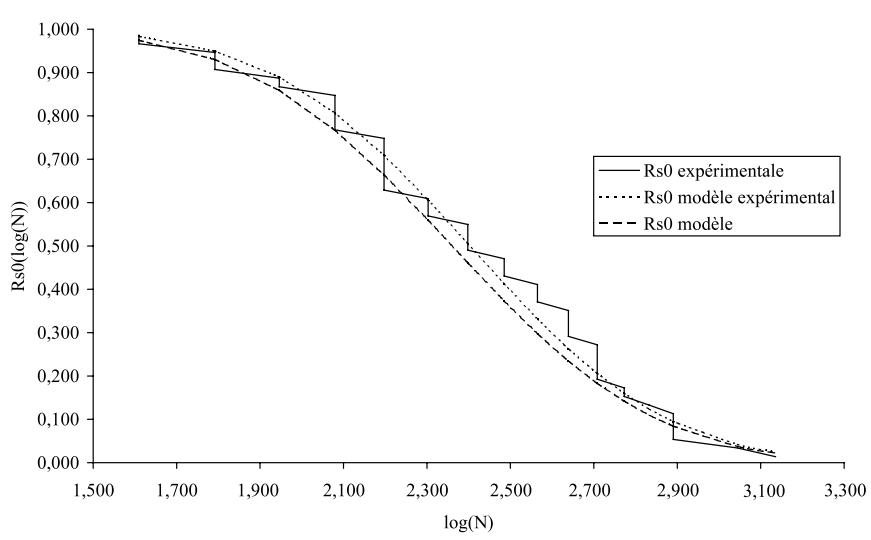

Fig. 13. Fonction fiabilité par un modèle SVA paramétrique.

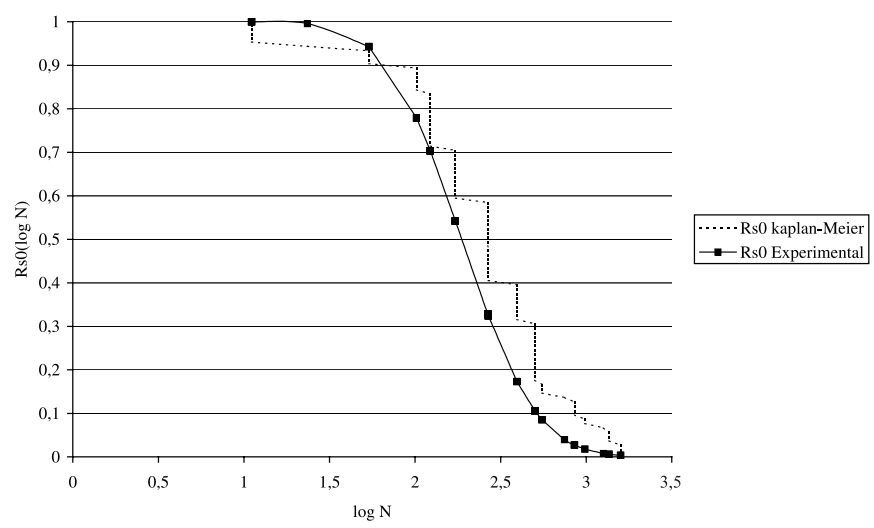

Fig. 14. Fonction de fiabilité par un modèle SVA nonparamétrique.

\section{a) Application du modèle SVA paramétrique}

En considérant une loi normale pour caractériser la fonction de fiabilité, nous obtenons par le maximum de vraisemblance (Éq. (39)) les résultats suivants :

$$
\begin{array}{cc}
\hat{\mu} & 2,36 \\
\hat{\sigma} & 0,385 \\
\hat{r} & 1,18
\end{array}
$$

De ces résultats, il est possible de définir la loi de fiabilité dans les conditions nominales (Fig. 14). 
Tableau 2. Résultats expérimentaux.

\begin{tabular}{|c|c|c|c|c|c|}
\hline$N-45^{\circ}$ & $\log \left(N-45^{\circ}\right)$ & $N-90^{\circ}$ & $\log \left(N-90^{\circ}\right)$ & $N 90^{\circ}+45^{\circ}$ & $\log \left(N 90^{\circ}-45^{\circ}\right)$ \\
\hline 5 & 1,609 & 4 & 1,386 & 4 & 1,386 \\
\hline 5 & 1,609 & 4 & 1,386 & 4 & 1,386 \\
\hline 6 & 1,792 & 4 & 1,386 & 4 & 1,386 \\
\hline 6 & 1,792 & 4 & 1,386 & 4 & 1,386 \\
\hline 6 & 1,792 & 4 & 1,386 & 4 & 1,386 \\
\hline 7 & 1,946 & 5 & 1,609 & 4 & 1,386 \\
\hline 7 & 1,946 & 5 & 1,609 & 5 & 1,609 \\
\hline 8 & 2,079 & 5 & 1,609 & 5 & 1,609 \\
\hline 8 & 2,079 & 5 & 1,609 & 5 & 1,609 \\
\hline 8 & 2,079 & 5 & 1,609 & 5 & 1,609 \\
\hline 8 & 2,079 & 5 & 1,609 & 5 & 1,609 \\
\hline 8 & 2,079 & 6 & 1,792 & 5 & 1,609 \\
\hline 9 & 2,197 & 6 & 1,792 & 6 & 1,792 \\
\hline 9 & 2,197 & 6 & 1,792 & 6 & 1,792 \\
\hline 9 & 2,197 & 6 & 1,792 & 6 & 1,792 \\
\hline 9 & 2,197 & 6 & 1,792 & 6 & 1,792 \\
\hline 9 & 2,197 & 6 & 1,792 & 7 & 1,946 \\
\hline 9 & 2,197 & 6 & 1,792 & 8 & 2,079 \\
\hline 9 & 2,197 & 6 & 1,792 & 8 & 2,079 \\
\hline 10 & 2,303 & 6 & 1,792 & 8 & 2,079 \\
\hline 10 & 2,303 & 6 & 1,792 & 8 & 2,079 \\
\hline 10 & 2,303 & 6 & 1,792 & 9 & 2,197 \\
\hline 11 & 2,398 & 6 & 1,792 & 9 & 2,197 \\
\hline 11 & 2,398 & 7 & 1,946 & 9 & 2,197 \\
\hline 11 & 2,398 & 7 & 1,946 & 9 & 2,197 \\
\hline 11 & 2,398 & 7 & 1,946 & 10 & 2,303 \\
\hline 12 & 2,485 & 7 & 1,946 & 10 & 2,303 \\
\hline 12 & 2,485 & 7 & 1,946 & 10 & 2,303 \\
\hline 12 & 2,485 & 7 & 1,946 & 10 & 2,303 \\
\hline 13 & 2,565 & 7 & 1,946 & 11 & 2,398 \\
\hline 13 & 2,565 & 7 & 1,946 & 11 & 2,398 \\
\hline 13 & 2,565 & 8 & 2,079 & 11 & 2,398 \\
\hline 14 & 2,639 & 8 & 2,079 & 11 & 2,398 \\
\hline 14 & 2,639 & 8 & 2,079 & 12 & 2,485 \\
\hline 14 & 2,639 & 8 & 2,079 & 12 & 2,485 \\
\hline 14 & 2,639 & 8 & 2,079 & 12 & 2,485 \\
\hline 15 & 2,708 & 8 & 2,079 & 12 & 2,485 \\
\hline 15 & 2,708 & 8 & 2,079 & 12 & 2,485 \\
\hline 15 & 2,708 & 8 & 2,079 & 13 & 2,565 \\
\hline 15 & 2,708 & 8 & 2,079 & 13 & 2,565 \\
\hline 15 & 2,708 & 9 & 2,197 & 13 & 2,565 \\
\hline 16 & 2,773 & 9 & 2,197 & 14 & 2,639 \\
\hline 16 & 2,773 & 9 & 2,197 & 14 & 2,639 \\
\hline 17 & 2,833 & 10 & 2,303 & 14 & 2,639 \\
\hline 18 & 2,890 & 11 & 2,398 & 15 & 2,708 \\
\hline 18 & 2,890 & 11 & 2,398 & 15 & 2,708 \\
\hline 18 & 2,890 & 12 & 2,485 & 15 & 2,708 \\
\hline 18 & 2,890 & 13 & 2,565 & 15 & 2,708 \\
\hline 21 & 3,045 & 13 & 2,565 & 16 & 2,773 \\
\hline 23 & 3,135 & 13 & 2,565 & 18 & 2,890 \\
\hline
\end{tabular}




\section{b) Application d'un modèle SVA non-paramétrique}

$\mathrm{Si}$ on applique le modèle non-paramétrique aux résultats de simulation du tableau 2 , il est possible de construire la loi de fiabilité dans les conditions nominales à l'aide de l'estimateur de Kaplan-Meier (Éq. (43)). Nous obtenons une estimation de $r$ par le maximum de vraisemblance (Éq. (44)) :

$$
\hat{r}=1,1944
$$

La loi de fiabilité construite par l'estimateur KaplanMeier est donnée par la figure 14.

\subsubsection{Analyse des résultats}

Nous constatons une bonne adéquation des modèles paramétriques et non-paramétriques avec les résultats de référence (essai à $45^{\circ}$ ). Ce plan d'essai est très séduisant car il nécessite une faible paramétrisation de la fonction $r$ qui peut être source d'erreur. On montre à travers cet exemple l'intérêt d'utiliser ce plan d'essai avec un endommagement préalable permettant d'obtenir de bon résultat.

\section{Conclusions}

Nous avons présenté une étude complète d'application du modèle standard de vie accélérée à la mécanique en considérant les estimations paramétrique, semi-paramétrique et non-paramétrique. Pour l'analyse, nous avons considéré le mode d'endommagement par fatigue. Ainsi, nous avons pu étudier 2 types de plan d'essai :

1. le premier consiste à déterminer les paramètres du modèle SVA à partir de résultats d'essais effectués uniquement dans des conditions sévérisées et de déduire par régression la loi de fiabilité en conditions nominales,

2. le deuxième permet de définir la loi de fiabilité dans les conditions nominales en procédant par un essai où au départ les composants sont testés dans des conditions sévérisés afin de provoquer une dégradation rapide et de finir l'essai dans les conditions nominales.

Les différents exemples ont montré l'adéquation du modèle SVA à la mécanique. Toutefois, le premier plan d'essai par régression nécessite une bonne connaissance de la fonction $r(s)$ ce qui n'est pas toujours évident surtout dans les cas où de nombreuses variables sont utilisées pour sévériser les essais. Aussi, le deuxième plan offre une réelle alternative en proposant une méthodologie où il n'est pas nécessaire de paramétriser la fonction $r(s)$ qui est la réelle difficulté dans une campagne d'essais accélérés.
Néanmoins, dans cette étude nous avons considéré que le paramètre de forme de la loi de fiabilité est constant quelque soit le niveau de stress. Or, en mécanique ce n'est pas toujours le cas. Notre étude se poursuit donc en intégrant ce dernier paramètre.

\section{Références}

[1] M.S. Phadke, Quality engineering using robust design, Prentice-Hall, 1989

[2] F. Guérin, B. Dumon, R. Hambli, Determining the shape parameter of a Weibull distribution from MechanicalDamage Model, Annual Reliability and Maintainability symposium, Proceedings, 2001, pp. 156-160

[3] F. Guérin, B. Dumon, R. Hambli, O. Tebbi, Accelerated Testing based on a Mechanical-Damage Model, Annual Reliability and Maintainability symposium, Proceedings, 2001, pp. $372-376$

[4] O. Tebbi, F. Guérin, B. Dumon, Reliability testing of Mechanical products-Application of statistical accelerated life testing models, 9th International Conference on Applications of Statistics and Probability in Civil Engineering, July 6-9, 2003, University of California, Berkeley

[5] W. Nelson, Accelerated Testing: Statistical Models, Test Plans and Data Analysis, Wiley Series in Probability and Mathematical Statistics, New York, 1990

[6] V. Bagdonavicius, M. Nikulin, On accelerated testing of systems, Europ. J. Diagnosis Safety Automation 5(3) (1995) 307-316

[7] J.H. Bompas-Smith, Mechanical Survival: the use of reliability data, Mc Graw Hill book, 1973

[8] V. Bagdonavicius, M. Nikulin, Transfer functional and semi-parametric regression, Biometrika V 84(2) (1997) $365-378$

[9] D. Kececioglu, M. Jiang, F.-B. Sun, A unified approach to random fatigue reliability quantification under random loading, Proc. IEEE Reliability and Maintenability, 1998

[10] V. Bagdonavicius, M. Nikulin, Semi-parametrics Models in Accelerated Life Testing, Queen's papers in pure and applied mathematics, Queen's University, Kingston, Ontario, Canada, 1995, p. 70

[11] Hank Caruso, Abhijit Dasgupta, A Fundamental Overview of Accelerated Testing Analytical Models, Proceedings Annual Reliability and Maintainability Symposium, 1998, pp. 389-393

[12] K. Devarajan, N. Ebrahimi, Non-parametric Approach to Accelerated Life Testing under Multiple Stress, Naval Research Logistics, John Wiley \& Sons, 1998, Inc 45, pp. 629-644

[13] R.L. Doyle, Mechanical Reliability, RAMS Tutorial Notes, 1991

[14] D.G. Ford, D.G. Graff, A.O. Payne, Some statistical aspects of fatigue life variation, Fatigue aircraft structure, Proc. 2nd ICAF Symposium, 1961, Paris, pp. $179-208$

[15] Pantelis Vassilious, Adamantios Mettas, Understanding Accelerated Life-Testing Analysis, Annual Reliability and Maintainability symposium, Tutorial Notes, 2001, pp. $1-21$ 
[16] H.-J. Shyur, E.A. Elsayed, J.T. Luxhoj, A general model for Accelerated Life Testing with time-dependent covariates, Naval Research Logistics 46 (1999) 303-321

[17] C. Zhang, Mechanical component lifetime estimation based on accelerated life testing with singurality extrapolation, Mechanical Systems and Signal Processing 16 (2002) 705-718

[18] W.J. Owen, W.J. Padgett, Accelerated test models for system strength based on Birnbaum-Saunders distributions, Lifetim Data Analysis, 5 (1998) 133-147

[19] P. O'Connor, Testing for reliability, Quality and Reliability Engineering international 19 (2003) 73-84

[20] Hoang Phan, Handbook of reliability engineering, Springer, 2003

[21] A.P. Basu, N. Ebrahimi, Non-parametric Accelerated Life Testing, IEEE Trans. Reliab. 31(5) (1982) 432-435
[22] V. Bagdonavicius, L. Gerville-Reache, V. Nikoulina, M. Nikoulin, Expériences accélérées : analyse statistique du modèle standard de vie accélérée, Revue de Statistique Appliquée, Vol. XLVIII, septembre 2000, 5-38

[23] V. Bagdonavicius, M. Nikulin, Mathematical models in the theory of accelerated experiments, 2001 World Scientific Publishing Co., pp. 271-303

[24] Christian Lalanne, Dommage par fatigue, Tome 4, Hermes Science Publications, Paris, 1999

[25] J. Lemaitre, J.L. Chaboche, Mécanique des matériaux solides, Dunod, Paris, 1996

[26] R.E. Little, J.C. Ekvall, Statistical Analysis of Fatigue Data, American Society for Testing and Materials, STP744, 1979

[27] J.E. Shigley, Mechanical Engineering Design, Mc Graw Hill, 1972

Retrouvez nos articles sur le site : www.edpsciences.org/meca 